\title{
Note on Binet's Inverse Factorial Series for $\mu(x)$.
}

\author{
By E. T. Copson.
}

Received and Read, 6th March, 19z5.

Binet * shewed that the function

$$
\mu(x)=\log \Gamma(x)-\left(x-\frac{1}{2}\right) \log x+x-\frac{1}{2} \log 2 \pi
$$

can be expanded as an inverse factorial series. This note furnishes a new and much simpler proof $\dagger$ of his result, based on a formula which is an analogue $\ddagger$ of the Binomial Theorem for factorials. This formula is that, if we denote by $[x]^{n}$ the ratio

$$
\Gamma(1+x) / \Gamma(1+x-n),
$$

then

$$
[x+h]^{m}=[x]^{m}+\left(\begin{array}{c}
m \\
1
\end{array}\right)[x]^{m-1}[h]^{1}+\left(\begin{array}{c}
m \\
2
\end{array}\right)[x]^{m-2}[h]^{2}+\ldots
$$

where $\left(\begin{array}{c}m \\ r\end{array}\right)$ denotes the coefficient of $x^{r}$ in the expansion of $(1+x)^{m}$. This formula is valid if $R(x+h+1)>0$. Putting $m=-1$ in this formula, we have, provided $R(x+t)>0$,

$$
1-\frac{x}{x+t}=\sum_{n=1}^{\infty}(-1)^{n+1}[x]^{-n}[t]^{n}
$$

If we denote by $\Sigma_{x}$ the ordinary finite-difference summation operator with respect to $x$, we easily see that, if $\varpi(x)$ is an arbitrary periodic function of period unity,

* Jourm. de lEEcole Polyt. 16 (1839), 123.

t The urual proof depends on expressing $\mu(x)$ as an integral. Soe NreLsEx : Handbuch der Theorie der Gammafunktionen; p. 284 et seq.

¥ This is merely the theorem that $F(a, b ; c ; 1)$ can be expressed as $\Gamma(c) \Gamma(c-a-b) / \Gamma(c-a) \Gamma(c-b)$ if $R(c-a-b)>0$. 


$$
\begin{aligned}
2 \mu(x)+\varpi(x) & =\Sigma_{x}\left\{2-x \log \frac{x}{x^{j}-1}-x \log \frac{x+1}{x}\right\}+\left\{1-x \log \frac{x}{x-1}\right\} \\
& =\Sigma_{x} \int_{-1}^{0}\left\{2-\frac{x}{x+t}-\frac{x}{x+t+1}\right\} d t+\int_{-1}^{0}\left\{1-\frac{x}{x+t}\right\} d t \\
& =\Sigma_{x} \int_{-1}^{0} \sum_{n=1}^{\infty}(-1)^{n+1}[x]^{-n}\left\{[t]^{n}+[t+1]^{n}\right\} d t \\
& +\int_{-1}^{0} \sum_{n=1}^{\infty}(-1)^{n+1}[x]^{-n}[t]^{n} d t \\
& =\Sigma_{x} \int_{-1}^{0} \sum_{n=2}^{\infty}(-1)^{n+1}[x]^{-n}\left\{[t]^{n}+[t+1]^{n}\right\} d t \\
& =\Sigma \int_{-1}^{0} \sum_{n=1}^{\infty}(-1)^{n}[x]^{-n+1}\left\{[t]^{n+1}+[t+1]^{n+1}\right\} d t \\
& +\int_{-1}^{0} \cdot \sum_{1}^{\infty}(-1)^{n+1}[x]^{-n}[t]^{n} d t .
\end{aligned}
$$

Now as $\Sigma_{x}$ is a finite difference operator, we can operate inside the signs of integration and summation, and so we obtain

$$
\begin{aligned}
2 \mu(x)+\varpi(x) & =\int_{-1}^{0} \sum_{n=1}^{\infty}(-1)^{n+1} \frac{1}{n}[x]^{-n}\left\{[t]^{n+1}+[t+1]^{n+1}+n[t]^{n}\right\} d t \\
& =\int_{-1}^{0}(2 t+1) \sum_{n=1}^{\infty}(-1)^{n+1} \frac{1}{n}[x]^{-n}[t]^{n} d t .
\end{aligned}
$$

Before we can integrate the right hand side term by term, we must investigate the convergence of the series occurring there.

Now Landau* has shewn that the inverse factorial series $\Omega(x)=\stackrel{\sum}{n=0}_{n}^{\infty} ! a_{n}[x-1]^{-\overline{n+1}}$ converges or diverges everywhere with the Dirichlet Series $\Psi(x)=\sum_{n=1}^{\infty} \frac{a_{n}}{n^{x}}$ whilst the binomial coefficient series $W(x)=\sum_{n=0}^{\infty} \frac{a_{n}}{n !}[x-1]^{n}$ converges or diverges every where with the Dirichlet Series $\sum_{n=1}^{\infty} \frac{(-1)^{n} a_{n}}{n^{x}}$. Using these results, which

* LAXDAO : Ḿnich Sitzungsberichle (1906) 36, 151-221. 
apply to convergence, uniform convergence and absolute convergence, we see that the series $\sum_{n=1}^{\infty}(-1)^{n+1} \frac{1}{n}[x]^{-n}[t]^{n}$ converges or diverges everywhere with the series $\sum_{n=1}^{\infty} \frac{1}{n^{x+t+2}}$. But if $t$ lies in the interval $(-1,0)$ this series converges uniformly with respect to $t$, provided that $R(x)>0$ Hence if $R(x)>0$, term by term integration is legitimate. By putting $t=-a$, and using the fact that $\mu(x) \rightarrow 0$ as $x \rightarrow+\infty$, we finally have

$$
\mu(x)=\sum_{n=1}^{\infty} \frac{[x]^{-n}}{2 n} \int_{0}^{1}(a+1)(a+2) \ldots(a+n-1)(2 a-1) a d a
$$

provided that $R(x)>0$. This is Binet's result. It should be noticed that the series converges nowhere on the line $R(x)=0$. 